Avaliação in silico do Potencial Inibitório da Luteolina em Dioclea virgata Frente à Enoil-ACP-Redutase de Plasmodium falciparum

\author{
Cardoso, A. R.; Brandão, H. N.; Santos Junior, M. C.; Pereira, L. R. M.; \\ Silva, D. A. A.; Alves, C. Q.*
}

Rev. Virtual Quim., 2019, 11 (2), 488-497. Data de publicação na Web: 29 de março de 2019

http://rvq.sbq.org.br

\title{
In silico Evaluation of the Inhibitory Potential of Luteolin in Dioclea virgata Against Enoil-ACP-Reductase of Plasmodium falciparum
}

Abstract: Malaria is a parasitic infection considered a serious global public health problem. Plasmodium falciparum is the causative agent of malaria that presents a greater adaptability by mutation causing resistance to antimalarial drugs. The use of plant material in the treatment of malaria aroused to search natural products as a source of molecules or inspiration for the development of new antimalarial drugs. Dioclea virgata was selected because of its antimalarial potential and for being a plant species present in the semiarid. The objective of this study was to identify flavonoids in $D$. virgata with potential inhibitory to the promising target, Enoyl-ACP Reductase, present in $P$. falciparum (PfENR) by virtual screening. Analysis of the extracts of leaves and stems from the $D$. virgata, by HPLCDAD allowed to identify the flavonoid luteolin in chloroform extract of leaves, which obtained an affinity energy value of $-32.03 \mathrm{Kcal} / \mathrm{mol}$ in relation to the enzyme. This negative value indicates that intermolecular interactions were formed easily.

Keywords: Malaria; luteolin; virtual screening; PfENR; Dioclea virgata.

\section{Resumo}

A malária é uma infecção parasitária considerada como um sério problema mundial de saúde pública. Plasmodium falciparum é o agente causador da malária, que apresenta elevada capacidade de adaptação por mutação, ocasionando resistência aos antimaláricos. A utilização de materiais vegetais no tratamento de malária possui grande valia para a pesquisa e desenvolvimento de novos antimaláricos. A Dioclea virgata foi escolhida para este estudo devido ao seu potencial antimalárico e por ser uma espécie vegetal presente no semiárido brasileiro. O objetivo do estudo foi identificar flavonoides na espécie $D$. virgata com potencial inibidor ao alvo promissor, Enoil-ACP Redutase, presente no $P$. falciparum (PfENR) por triagem virtual. A análise dos extratos de folhas e caule de $D$. virgata por CLAEDAD possibilitou identificar o flavonoide luteolina no extrato clorofórmico das folhas, que obteve valor de energia de afinidade de $-32.03 \mathrm{Kcal} / \mathrm{mol}$ frente à enzima. Esse valor negativo indica que as interações intermoleculares foram formadas com maior facilidade.

Palavras-chave: Malária; flavonoides; triagem virtual; PfENR. Dioclea virgata.

\footnotetext{
* Universidade Estadual de Feira de Santana, Departamento de Saúde, Laboratório de Modelagem Molecular, Avenida Transnordestina s/n, Bairro Novo Horizonte, CEP 44036-900, Feira de Santana-BA, Brasil.

Mcleiroz@gmail.com

DOI: $10.21577 / 1984-6835.20190037$
} 


\section{Avaliação in silico do Potencial Inibitório da Luteolina em Dioclea virgata Frente à Enoil-ACP-Redutase de Plasmodium falciparum}

\section{Andressa R. Cardoso, Hugo N. Brandão, Manoelito C. dos Santos Junior, Leane Rodrigues Moreira Pereira, Dayse A. A. Silva, Clayton Q. Alves*}

Universidade Estadual de Feira de Santana, Avenida Transnordestina s/n, Bairro Novo Horizonte, CEP 44036-900, Feira de Santana-BA, Brasil.

*cleiroz@gmail.com

Recebido em 6 de março de 2019. Aceito para publicação em 6 de março de 2019

\section{Introdução}

\section{Metodologia}

2.1. Coleta e identificação da espécie vegetal

2.2. Obtenção dos extratos de Dioclea virgata

2.3. Análise dos extratos por CLAE-DAD

2.4. Estudos de acoplamento molecular

\section{Resultados e Discussão}

3.1. Obtenção dos extratos

3.2. Identificação de flavonoides em Dioclea virgata por CLAE-DAD

3.3. Acoplamento molecular

3.4. Análise das interações intermoleculares

\section{Conclusão}

\section{Introdução}

As doenças parasitárias acometem uma parcela significativa da população mundial, provocando mortes e exercendo influência limitante na qualidade de vida e no desenvolvimento dos países endêmicos. ${ }^{1} \mathrm{~A}$ malária é uma das infecções parasitárias que causa maior preocupação e pode ser considerada um dos mais sérios problemas mundiais de saúde pública, visto que provoca inúmeros casos de morbidade e mortalidade. Estima-se que 3,2 bilhões de pessoas correm o risco de estarem infectados com parasitas do gênero Plasmodium, agente causador da malária. $^{2}$

Os mosquitos do gênero Anopheles infectados por protozoários do gênero Plasmodium são responsáveis pela transmissão da malária em seres humanos. Apenas cinco espécies promovem essa infecção: Plasmodium vivax, $P$. ovale, $P$. malariae, $P$. knowlesi e $P$. falciparum, sendo, o 
P. falciparum o mais perigoso por causar a malária cerebral, forma mais grave que na maioria dos casos leva à morte. ${ }^{3}$

Embora os fármacos antimaláricos desempenhem um papel central no controle e eliminação da doença, a sua atividade contra o Plasmodium sp. mostrou eficácia reduzida, devido à resistência que os parasitas vêm desenvolvendo. O Plasmodium falciparum, é um dos agentes causadores da malária que apresenta alta capacidade de adaptação por mutação, ocasionando resistência aos antimaláricos. Isto faz com que o desenvolvimento de novos agentes terapêuticos antimaláricos seja um assunto de grande interesse para a saúde pública. ${ }^{4,5}$

A síntese de ácido graxo tipo II, do inglês "Fatty Acid Synthase II" (FAS II) pelo P. falciparum é de grande relevância para a pesquisa de antimaláricos. Isso porque as enzimas envolvidas nessa biossíntese estão ausentes nos seres humanos, e por esse motivo são alvos potenciais para o desenvolvimento de novos fármacos. A EnoilACP Redutase (ENR) é uma enzima presente na rota FAS II, ${ }^{6}$ uma etapa essencial no processo, inclusive para o desenvolvimento do plasmódio. Esse processo transforma importantes precursores em componentes celulares, incluindo fosfolipídeos, lipoproteínas, lipopolissacarídeos e ácidos micólicos. Além disso, essa biossíntese é uma importante fonte de energia, que desempenha um papel fundamental na transdução de sinal, na acilação de proteínas, bem como são necessários para o crescimento, diferenciação e homeostase do P. falciparum. ${ }^{7,8}$ Dessa forma, ao inibir a síntese dos ácidos graxos por inibição da enzima ENR promove-se a morte do parasita, sendo, portanto, um alvo adequado para o desenvolvimento de novos antimicrobianos e antiprotozoários, incluindo antimaláricos. ${ }^{9}$

As plantas medicinais são uma fonte potencial de novos fármacos contra a malária, tendo em vista, a presença de uma variedade de metabólitos com diversidade estrutural e atividade farmacológica. ${ }^{10}$ Estudos mostram que a utilização de materiais vegetais no tratamento da malária é promissora, tendo em vista relatos do uso popular nas folhas de Dioclea virgata no tratamento desta enfermidade. ${ }^{11}$ Além disso, foram identificados nessa espécie, que tem ocorrência tanto na Amazônia quanto no semiárido baiano, vários flavonoides, como a 7-hidroxi-6-metoxiflavona, a 3,5-dihidroxi-4'metoxi-isoflavona e a 7-hidroxi-6metoxiflavanona. ${ }^{12}$

Assim, a espécie $D$. virgata foi escolhida para este estudo devido a seu potencial antimalárico juntamente com o fato de ser uma espécie vegetal presente no semiárido, rica em metabólitos da classe dos flavonoides.

Dessa forma, o presente estudo justifica-se pela necessidade na busca de moléculas para o tratamento da malária, bem como pelo potencial antimalárico apresentado por alguns flavonoides ao inibir o promissor alvo, Enoil-ACP-Redutase, presente na etapa limitante da biossíntese do ácido graxo do tipo II (FAS II) do Plasmodium falciparum. Visando tal objeto, foi realizado um estudo fitoquímico em partes da Dioclea virgata, e os flavonoides identificados foram avaliados in silico quanto à sua afinidade molecular frente à Enoil-ACPRedutase.

\section{Metodologia}

\subsection{Coleta e identificação da espécie vegetal}

As folhas e cascas do caule de $D$. virgata (Rich.) Amsh (Leguminosae) foram coletadas na Universidade Federal da Bahia (UFBA), campus Ondina, município de Salvador, Bahia, em novembro de 2014. A identificação botânica da espécie foi realizada pelo botânico Luciano Paganucci de Queiroz, comparada com exsicata depositada no Herbário da Universidade Estadual de Feira de Santana, sob o número de exsicata HUEFS 115.979. 


\subsection{Obtenção dos extratos de $D$. virgata}

O material vegetal coletado foi submetido à secagem em estufa com renovação de ar a $40 \pm 3^{\circ} \mathrm{C}$, durante cinco dias até alcançar peso constante. As folhas e cascas do caule secos foram triturados em liquidificador industrial. A extração das folhas de $D$. virgata foi realizada através do método de maceração utilizando como solvente extrator o metanol. Um total de cinco macerações foi realizada, com a renovação do solvente extrator a cada três dias. Os filtrados obtidos em cada uma das cinco etapas de maceração foram reunidos e concentrados em evaporador rotatório com banho à temperatura de $60 \pm 1{ }^{\circ} \mathrm{C}$. O material concentrado foi seco em capela à temperatura ambiente, com o objetivo de obter o extrato bruto seco das folhas, uma alíquota deste extrato foi separada e a parte restante submetida à partição líquido-líquido.

O extrato bruto das cascas do caule de $D$. virgata foi obtido através do método de extração por refluxo, utilizando como solvente extrator o metanol. A solução obtida foi concentrada em evaporador rotatório com banho a temperatura de $60 \pm 1^{\circ} \mathrm{C}$. O material concentrado foi seco em capela à temperatura ambiente, com o objetivo de obter o extrato bruto seco das cascas do caule. Uma alíquota deste extrato foi separada e a parte restante submetida à partição líquidolíquido.

A partição líquido-líquido dos extratos bruto de folhas e das cascas do caule de $D$. virgata foi realizada utilizando solventes orgânicos em ordem crescente de polaridade: hexano, clorofórmio e acetato de etila. Os extratos hexânico, clorofórmico e acetato de etila foram filtrados e concentrados em evaporador rotatório, secos em capela à temperatura ambiente.

\subsection{Análise dos extratos por CLAE-DAD}

Os extratos secos (bruto, hexânico, clorofórmio e acetato de etila) obtidos de folhas e cascas do caule foram submetidos à análise por CLAE-DAD com o intuito de detectar a presença de flavonoides. A identificação foi realizada através da comparação com padrões comerciais. Os padrões analisados foram canferol, canferide, rutina, quercetina e luteolina, todos obtidos da Sigma-Aldrich ${ }^{\circledR}$.

As análises cromatográficas foram realizadas através do cromatógrafo Varian com sistema HPLC Workstation, consistindo de bomba Varian Polaris, detector de arranjo de diodo (DAD) Varian ProStar e injetor manual. Para a separação cromatográfica utilizou-se coluna LiChroCART Purospher Star ${ }^{\circledR}$ RP18-e $(75 \mathrm{~mm} \times 4 \mathrm{~mm})(3 \mu \mathrm{m})$ (Merck, Darmastad, Germany) combinada com précoluna LiChroCART 4-4 LiChrospher 100RP18 $(5 \mu \mathrm{m})$ da Merck. As condições cromatográficas incluíram: volume de injeção de $20 \mu \mathrm{L}$, faixa de comprimento de onda de $210-400 \mathrm{~nm}$, com aquisição em $280 \mathrm{~nm}$.

O gradiente de eluição utilizado para análise dos extratos consistiu de solução de ácido acético $0,7 \%$ (fase $A$ ) e acetonitrila (MeCN) com solução de ácido acético 0,7 \% na proporção de 8:2 (fase B) (Tabela 1), com fluxo $1,0 \mathrm{~mL} / \mathrm{min}$. Para as análises em CLAE foram usados solventes de grau cromatográfico de pureza (Merck ${ }^{\circledR}$ e Vetec $^{\circledR}$ ) e água purificada em sistema Milli- ${ }^{\circledR}$. 
Tabela 1- Gradiente de eluição da análise por CLAE-DAD

\begin{tabular}{ccc}
\hline Tempo (min) & Fase A (\%) & Fase B (\%) \\
\hline $0: 00$ & 95 & 5 \\
$1: 00$ & 95 & 5 \\
$5: 00$ & 90 & 10 \\
$12: 00$ & 83 & 17 \\
$30: 00$ & 20 & 80 \\
$30: 01$ & 0 & 100 \\
$35: 00$ & 0 & 100 \\
$38: 00$ & 95 & 5 \\
$40: 00$ & 95 & 5 \\
\hline
\end{tabular}

Fonte: Autoria própria, 2015

As soluções dos extratos de Diocle avirgata para análise em CLAE-DAD foram preparadas com a fase orgânica (fase B) em concentração de $10 \mathrm{mg} / \mathrm{mL}$, filtradas em cartucho de extração de fase sólida C18 (Cleanert ${ }^{\circledR}$ ) e filtro de membrana de éster de celulose 0,45 $\mu \mathrm{m}$ (Millipore ${ }^{\circledR}$ ). As soluções dos padrões analisados foram feitas em concentração de $1 \mathrm{mg} / \mathrm{mL}$, utilizando a fase orgânica $B$, essas soluções foram filtradas apenas em membrana de éster de celulose $0,45 \mu \mathrm{m}$ (Millipore ${ }^{\circledR}$ ).

\subsection{Estudos de acoplamento molecular}

A estrutura 3D de Enoil-ACP-Redutase (PDB: 3LTO) foi obtida do banco de estruturas macromoleculares Protein Data Bank. ${ }^{13} \mathrm{O}$ preparo do alvo foi realizado no programa Chimera 1.10.1, com isso, os átomos de hidrogênio e cargas Gasteiger-Huckel foram adicionados.

O acoplamento molecular foi realizado no programa DOCK 6.5 utilizando a função de pontuação GridScore. ${ }^{14}$ A delimitação do espaço de acoplamento molecular foi realizada por meio dos programas acessórios do DOCK 6.5. Através da superfície molecular de acesso ao solvente do receptor gerada pelo programa acessório $\mathrm{DMS},{ }^{15}$ foi construída a imagem negativa da superfície molecular do sítio ativo (localizada a partir do ligante cristalográfico) pelos programas SPHGEN e SPHERE_SELECTOR. ${ }^{16}$ As propriedades moleculares para a região de acoplamento molecular foram calculadas pelo programa GRID em sua configuração padrão. ${ }^{17,18} \mathrm{O}$ reacoplamento foi realizado com o intuito de avaliar a capacidade de predição correta das poses pelos programas de acoplamento molecular. Essa avaliação comparou a orientação e conformação já conhecidas do ligante cristalográfico, com a pose obtida do cálculo de acoplamento molecular. Os resultados foram avaliados com base no valor do desvio médio quadrático (RMSD). ${ }^{19}$

\section{Resultados e Discussão}

\subsection{Obtenção dos extratos}

Após os processos de secagem e pulverização, adquiriu-se uma massa de $1007,35 \mathrm{~g}$ de pó das folhas e $1890,2 \mathrm{~g}$ para as cascas do caule da espécie $D$. virgata. 0 pó obtido foi submetido ao processo de extração por maceração utilizando metanol como solvente extrator e, após o processo de secagem, obteve-se o equivalente a $72,66 \mathrm{~g}$ de 
extrato bruto seco para as folhas e $107,05 \mathrm{~g}$ para o caule, alcançando rendimento de 7,21 $\%$ e $5,66 \%$, respectivamente.

Para obtenção das frações semipurificadas, foi realizada a partição líquido-líquido com os extratos de $D$. virgata, empregando solventes orgânicos de polaridade crescente: hexano, clorofórmio e acetato de etila, implicando na obtenção das frações hexânica $(7,03 \mathrm{~g})$, clorofómica $(3,74 \mathrm{~g})$ e acetato de etila $(1,28 \mathrm{~g})$ das folhas e hexânica $(1,68 \mathrm{~g})$, clorofómica $(3,69 \mathrm{~g})$ e acetato de etila $(4,25 \mathrm{~g})$ do calule de D. virgata.

3.2. Identificação de flavonoides em $D$. virgata por CLAE-DAD

A análise dos extratos (bruto, hexânico, clorofórmico e acetato de etila) de folhas e cascas do caule de D. virgata, por CLAE-DAD possibilitou avaliar os flavonoides: canferol, canferide, rutina, quercetina e luteolina, através da comparação dos cromatogramas e espectros de UV dos extratos analisados com os padrões de flavonoides. Entretanto, a presença da luteolina foi observada somente no extrato clorofórmico de folhas de $D$. virgata.

Os tempos de retenção (tR) dos picos referentes à luteolina foram comparados nos cromatogramas do padrão (tR: 20,577min.) e do extrato clorofórmio de folhas (tR: 20,695 min.). As Figuras 1 e 2 mostram os cromatogramas e espectros de UV do padrão luteolina e do extrato clorofórmio das folhas de $D$. virgata, respectivamente. Os picos não identificados, por ausência de padrão específico, também apresentam características de substâncias flavonoídicas em função dos seus espectros de UV.

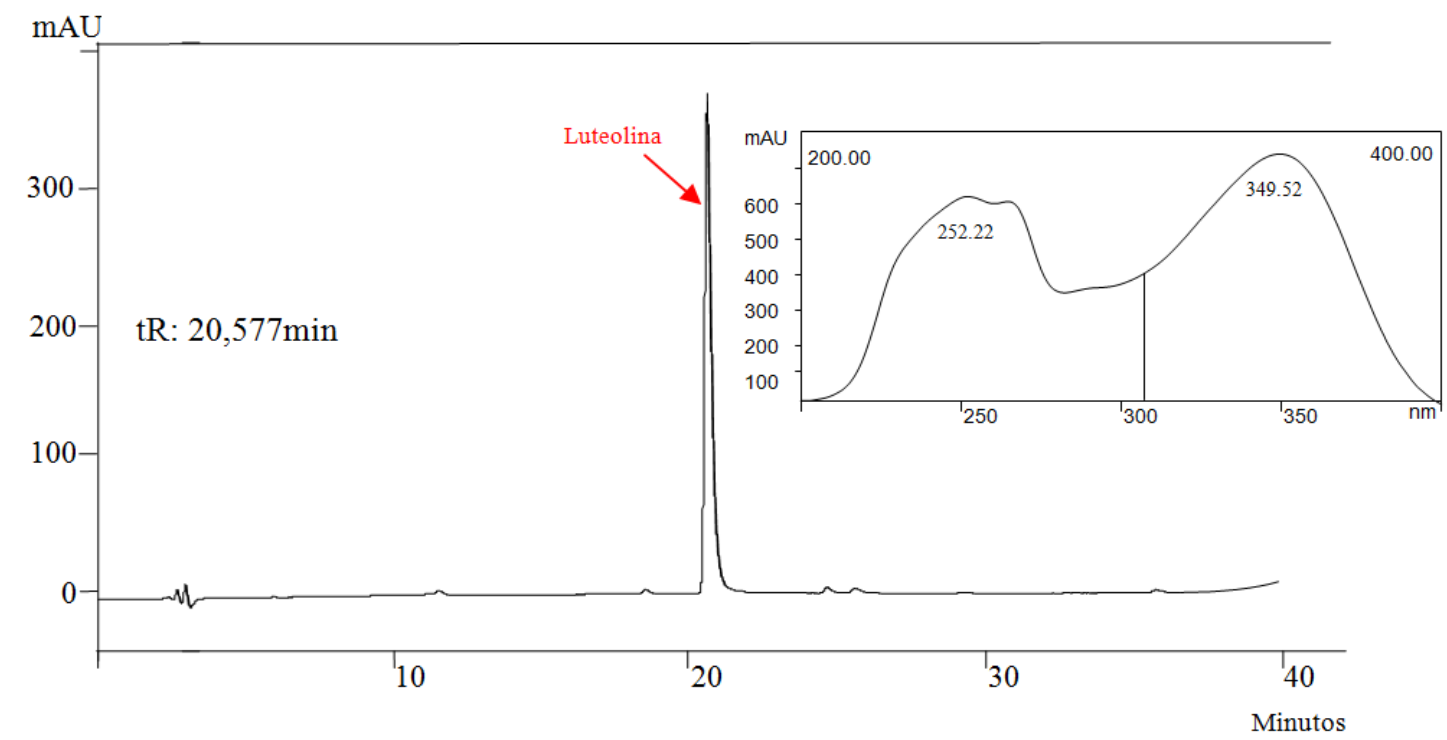

Figura 1. Cromatograma e espectro de UV do padrão Luteolina obtido da Sigma-Aldrich 


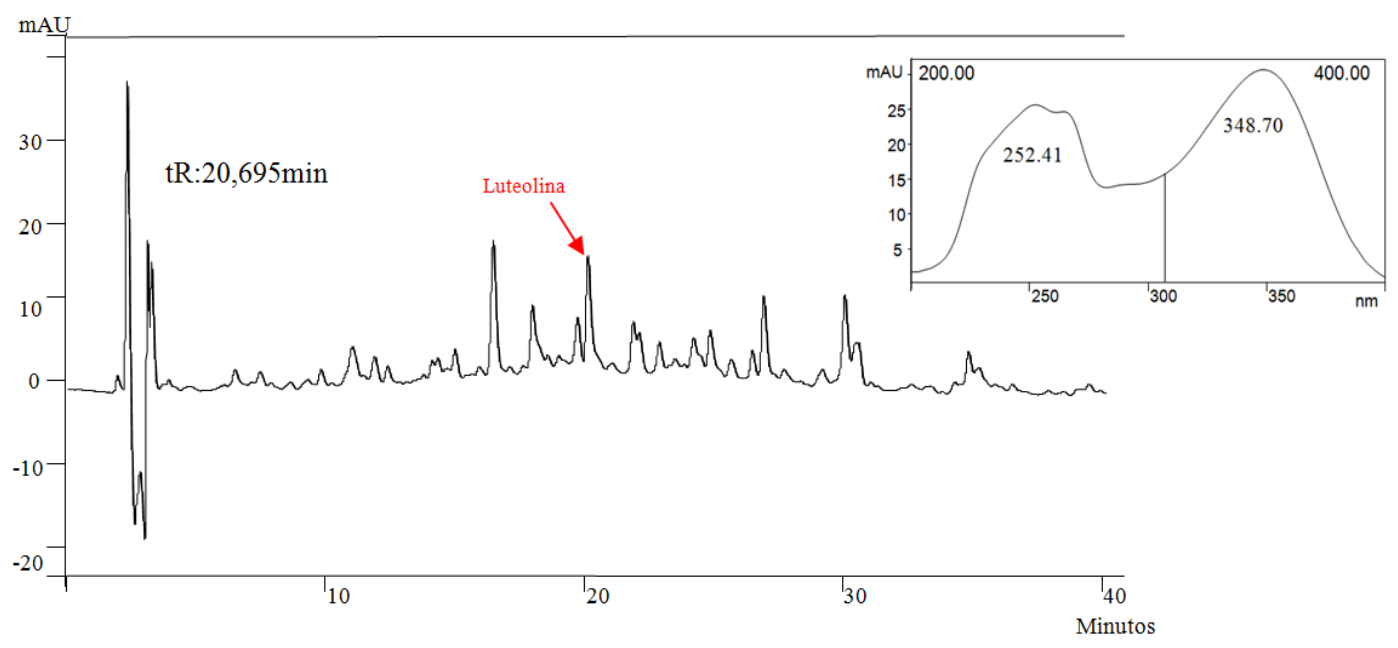

Figura 2. Cromatograma e espectro de UV dafração clorofórmica das folhas de $D$. virgata

\subsection{Acoplamento molecular}

O resultado obtido do reacoplamento molecular apresentou um valor de $\mathrm{RMSD}=0,54 \hat{A}$ (Figura 3). Foi possível inferir que o programa DOCK 6.5 obteve sucesso na orientação do ligante obtido pelo cálculo de acoplamento, comparado com a pose original do ligante cristalográfico, visto que o RMSD estava abaixo do valor limite de $2 \AA ̊$.

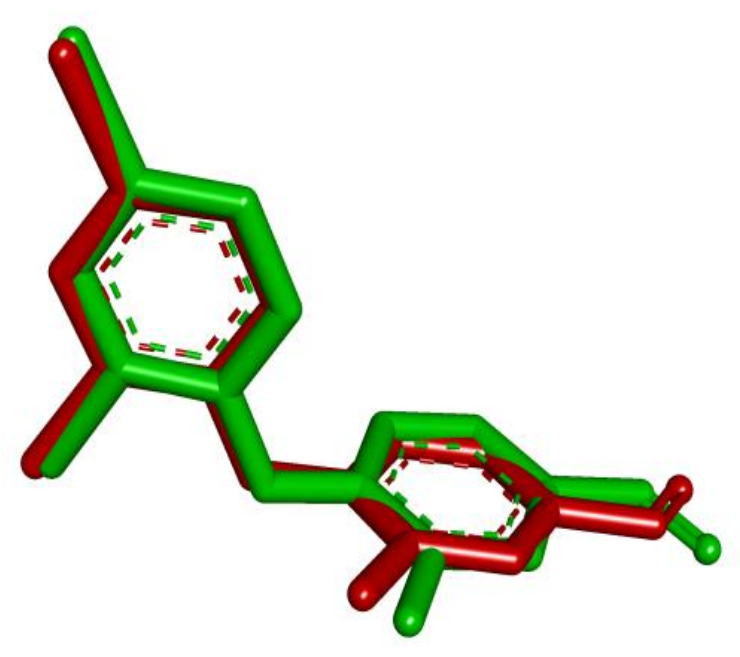

Figura 3. Sobreposição de duas poses do ligante cristalográfico 4-(2,4-diclorofenoxi)-3hidroxibenzaldeído. Estrutura cristalográfica do ligante (vermelho) e a estrutura da pose após o acoplamento molecular (verde) com o PfENR utilizando o programa DOCK6.5

O GridScore aplica o método sistêmico, com função de pontuação baseados no campo de força de mecânica molecular que quantifica a soma das energias de interação receptor-ligante e energia interna do ligante envolvido no processo de reconhecimento molecular. ${ }^{20,21}$
Utilizando a função GridScore, foi possível observar que a luteolina apresentou valor de energia de afinidade de $-32.03 \mathrm{Kcal} / \mathrm{mol}$. Valores negativos indicam que as interações intermoleculares são formadas mais facilmente. 


$\begin{array}{cl}\text { 3.4. Análise das interações } & \begin{array}{l}\text { Plasmodium falciparum (Figura 4), em que a } \\ \text { luteolina apresentou, com o núcleo } \\ \text { intermoleculares }\end{array} \\ \begin{array}{l}\text { flavonoídico, interações de hidrogênio } \\ \text { aceptora com a hidroxila da cadeia lateral de }\end{array} \\ \begin{array}{l}\text { Tyr277, além de interações hidrofóbicas com } \\ \text { após o acoplamento molecular, foram }\end{array} \\ \begin{aligned} \text { observadas as interações intermoleculares da laterais da Ala319 e lle323. } \\ \text { luteolina frente à Enoil-ACP-Redutase de }\end{aligned}\end{array}$

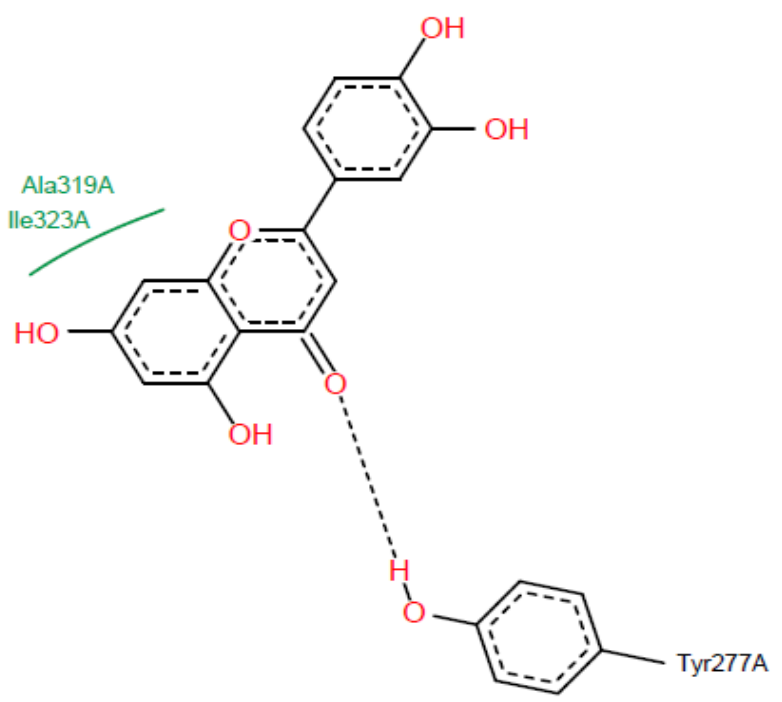

Figura 4- Mapa em 2D das interações intermoleculares de Luteolina - PfENR

\footnotetext{
Estudos da interação do complexo ternário PfENR-NAD+-triclosan mostram a presença de uma bolsa hidrofóbica, ou seja, uma região com propriedades de interação altamente hidrofóbicas formada pelos resíduos Tyr277, Tyr267, Gly313, Pro314, Ile323, Phe368, lle369, Ala372, Val274, Phe325, Ile326, Val222, Ala319, Ala320, Ile373, Ala217, Asn218, Ala219, Met281. ${ }^{22-24}$ No presente estudo foi possível encontrar algumas das interações hidrofóbicas descritas anteriormente, com os resíduos Ala319, lle323. Além das regiões de interações hidrofóbicas que caracterizam o sítio catalítico da enzima pfENR foram descritas na literatura a presença de interação de hidrogênio com a Tyr277, ${ }^{25}$ a qual pode ser observada na Figura 4. As interações identificadas favorecem uma atividade inibitória da enzima, tendo em vista que o Triclosan, substância com atividade inibitória comprovada, frente PfENR, também apresentou essas interações em estudos anteriores. ${ }^{22-24}$
}

\section{Conclusão}

Os resultados obtidos mostram que a energia de afinidade está relacionada ao somatório das interações de hidrogênio e hidrofóbicas, fato este reforçado pela literatura disponível sobre a inibição da PfENR. Além disso, foi possível observar a importância do núcleo flavonoídico para interações com a PfENR. Sendo assim, os valores negativos de afinidade sugerem que essas interações são favoráveis, corroborando com o potencial inibitório da luteolina presente em $D$. virgata.

\section{Agradecimentos}

Os autores agradecem ao Conselho Nacional de Desenvolvimento Científico e 
Tecnológico (CNPq) e à Fundação de Amparo à Pesquisa da Bahia (FAPESB) pelo suporte financeiro e bolsas concedidas.

\section{Referências Bibliográficas}

${ }^{1}$ França, T. C. C.; dos Santos, M. G.; FigueroaVillar, J. D. Malária: Aspectos Históricos e Quimioterapia. Química Nova 2008, 31, 1271. [CrossRef]

${ }^{2}$ OMS. World Malaria Report, Organização Mundial de Saúde 2014, 2. [Link]

${ }^{3}$ White, N. J.; Pukrittayakamee, S.; Hien, T. T.; Faiz, M. A.; Mokuolu, O. A.; Dondorp, A. M. Malaria Seminar 2014, 383, 723. [CrossRef]

${ }^{4}$ Sá, M. S.; Tese de Doutorado, Fundação Oswaldo Cruz, Brasil, 2011. [Link]

${ }^{5}$ Tasdemir, D.; Lack, G.; Brun, R.; Ruedi, P.; Scapozza, L.; Perozzo, R. Inhibition of Plasmodium falciparum Fatty Acid Biosynthesis: Evaluation of Fab G, Fab Z, and Fab I as Drug Targets for Flavonoids. Journal of Medicinal Chemistry 2006, 49, 3345. [CrossRef] [Pubmed]

${ }^{6}$ Muench, S. P.; Rafferty, J. B.; Mcleod, R.; Rice, D. W.; Prigge, S. T. Expression, purification and crystallization of the Plasmodium falciparum enoyl reductase. Acta Crystallographica 2003, 59, 1246. [CrossRef] [Pubmed]

${ }^{7}$ Andrade, C. H.; Pasqualoto, K. F. M.; Zaim, M. H.; Ferreira, E. I. Abordagem racional no planejamento de novos tuberculostáticos: Inibidores da InhA, enoil-ACP redutase do $M$. tuberculosis. Revista Brasileira de Ciências Farmacêuticas 2008, 44, 167. [CrossRef]

${ }^{8}$ Tasdemir, D. Type II fatty acid biosynthesis, a new approach in antimalarial Natural product discovery. Phytochemistry Reviews 2006, 5, 99. [CrossRef]

${ }^{9}$ Kapoor, N.; Banerjee, T.; Babu, P.; Maity, K.; Surolia, N.; Surolia, A. Design, development, synthesis, and docking analysis of 2'substituted triclosan analogs as inhibitors for Plasmodium falciparum Enoyl-ACP reductase. Life 2009, 61, 1083. [CrossRef] [Pubmed]
${ }^{10}$ Ntie-kang, F.; Onguéne, P. A.; Lifongo, L. L.; Ndom, J. C.; Sippl, W.; Mbaze, L. M. The potencial of anti-malarial compounds derived from African medicinal plants, part II: a pharmacological evaluation of non-alkaloids and non-terpenoids. Malaria Journa 12014, 13, 81. [CrossRef]

${ }^{11}$ Agra M. F., de Freitas P. F., Barbosa-Filho J. $M$. Sinopse das plantas conhecidas como medicinais e venenosas no nordeste do Brasil. Revista Brasileira de Farmacognosia 2007, 17, 114. [CrossRef]

${ }^{12}$ Alves, C. Q, David, J. M., David. J. P, Kijjoa, A., Flavonoids and other compounds from Dioclea virgate (Rich) Amsh. Biochemical Systematics and Ecology 2018, 78, 43. [CrossRef]

${ }^{13}$ Berman, H. M.; Westbrook, J.; Feng, Z.; Gilliland, G.; Bhat, T. N.; Weissig, H.; Shindyalov, I. N.; Bourne, P. E. The Protein Data Bank. Nucleic Acids Research 2000, 28, 235. [CrossRef] [Pubmed]

${ }^{14}$ Lang, P. T.; Allen, W. J.; Balius, T.; Brozell, S.; Fochtman, B.; Jiang, L.; McGee Jr, T. D.; Moustakas, D.; Mukherjee, S.; Prentis, L.; Singleton, C.; Telehany, S.; Zhou, Y.; Rizzo, R.; Case, D.; Shoichet, B.; Kuntz, I. DOCK 6.8 Users Manual. Regents of the University of California, 2017. [Link]

${ }^{15}$ Ferrin, T. E.; Huang, C.C; Jarvis, L. E.; Langridge, R. The MIDAS display system. Journal of Molecular Graphics 1988, 6, 13. [CrossRef]

${ }^{16}$ Kuntz, I. D.; Blaney, J. M.; Oatley, S. J.; Langridge, R.; Ferrin, T. E. A geometric approach to macromolecule-ligand interactions. Journal Molecular Biological 1982, 161, 269. [CrossRef] [Pubmed]

${ }^{17}$ Meng, E. C.; Shoichet, B.K.; Kuntz, I.D. Automated docking with grid-based energy evaluation. Journal of Computational Chemistry 1992, 13, 505. [CrossRef]

${ }^{18}$ Shoichet, B. K.; Bodian, D. L.; Kuntz, I. D. Molecular docking using shape descriptors. Journal Computational Chemistry 1992, 13, 380. [CrossRef] 
${ }^{19}$ Brozell, S.R.; Mukherjee, S.; Balius, T. E.; Roe, D. R.; Case, D. A.; Rizzo, R. C. Evaluation of DOCK 6 as a pose generation and database enrichment tool. Journal of Computer-Aided Molecular Design 2012, 26, 749. [CrossRef] [Pubmed]

${ }^{20}$ Andricopulo, A. D.; Salum, L. B.; Abraham, D.J. Structure-based drug design strategies in medicinal chemistry. Current Topics in Medicinal Chemistry 2009, 9, 771. [CrossRef] [Pubmed]

${ }^{21}$ Honório, K. M.; Silva, A. B. F. Forças intermoleculares e a interação fármacoreceptor. In: Montanari, C. A., Química Medicinal: métodos e fundamentos em planejamentos de fármacos, EDUSP: São Paulo, 2011. [Link]

${ }^{22}$ Freundlich, J. S.; Wang, F.; Tsai, H. C.; Kuo, M.; Shieh, H. M.; Anderson, J. W.; Nkrumah, L. J.; Valderramos, J. C.; Yu, M.; Kumar, T. R. S.; Valderramos, S. G.; Jr. Jacobs, W. R.; Schiehser, G. A.; Jacobus, D. P.; Fidock, D. A.; Sacchettini, J. C. X-ray Structural Analysis of Plasmodium falciparum Enoyl Acyl Carrier Protein Reductase as a Pathway toward the
Optimization of Triclosan Antimalarial Efficacy. The Journal of Biological Chemistry 2007, 282, 25436. [CrossRef] [Pubmed]

${ }^{23}$ Maity, K.; Bhargav, S. P.; Sankaran, B.; Surolia, N.; Surolia, A.; Suguna, K. X-ray crystallographic Analysis of the Complexes of Enoyl Acyl Carrier Protein Reductase of Plasmodium falciparum with Triclosan Variants to Elucidate the Importance of Different Functional Groups in Enzyme Inhibition. Life 2010, 62, 467. [CrossRef] [Pubmed]

${ }^{24}$ Stewart, M. J.; Parikh, S.; Xiao, G.; Tonge, P. J.; Kisker, C. Structural Basis and Mechanism of Enoyl Reductase inhibition by Triclosan. Journal the Molecular Biological 1999, 290, 859. [CrossRef] [Pubmed]

${ }^{25}$ Frecer, V.; Megnassan, E.; Miertus, S. Design and in silico screening of combinatorial library of antimalarial analogs of triclosan inhibiting Plasmodium falciparum Enoyl-acyl carrier protein reductase. European Journal of Medicinal Chemistry 2009, 44, 3009. [CrossRef] [Pubmed] 